\title{
Enhancing Visibility of the Hypopharyngeal- Esophageal Region by Introduction of a Physiological Eructation Maneuver
}

\author{
Svetlana Politz ${ }^{a}$ Martin Wagner ${ }^{b}$ Degenhart Schwub ${ }^{c}$ \\ Sandra Cattenberg ${ }^{a}$ Robert Thurnheer ${ }^{d}$ Ludwig Schelosky $^{a}$ \\ aDivision of Neurology, Kantonsspital Münsterlingen, Münsterlingen, Switzerland; \\ bSchön Klinik Bad Aibling, Bad Aibling, Germany; ' ${ }^{\mathrm{C} D i v i s i o n}$ of Otolaryngology, \\ Kantonsspital Münsterlingen, Münsterlingen, Switzerland; d Division of Pneumology, \\ Kantonsspital Münsterlingen, Münsterlingen, Switzerland
}

\section{What Is It about?}

- The hypopharyngeal-esophageal region (HER) at a glance: Better insight with a "burp."

- The eructation method was used during flexible transnasal endoscopy to obtain a view of the entire hypopharynx region as well as the upper esophageal sphincter. Frame-grabbed pictures were analyzed following the intervention. In a group out of 20 volunteers, 16 (80\%) were able to volitionally induce an eructation.

- In $13(81 \%)$ of the 16 subjects, the upper esophageal sphincter was made visible (partially or fully opened). The method has potential for early detection of cancer in the otherwise concealed hypopharyngeal area.

\section{Keywords}

Diagnostic methods · Endoscopic procedures · Hypopharyngeal cancer · Hypopharynx

\begin{abstract}
Background: In routine medical examination, the hypopharyngeal-esophageal area (HER) is difficult to assess due to its position and anatomical complexity. The purpose of this study was to evaluate the feasibility of a volitional eructation maneuver during transnasal flexible laryngoscopy and its influence on the visibility of the HER. Methods: Twenty healthy volunteers underwent flexible laryngoscopy. Once the larynx was freely visible during laryngoscopy, the subjects were asked to trigger a "burp." The volitional belching during the study was assisted by drinking a carbonated cold drink. The triggered relaxation of the upper esophageal sphincter along with the widening of the hypopharynx region was recorded and subsequently analyzed frame by frame. Results: Out of 20 volunteers, 16 (80\%) were able to volitionally induce an eructation. Significant widening of the hypopharynx region up to the 
relaxant upper esophageal sphincter could be recorded. The structures were clearly visible in the offline analysis. In 13 (81\%) of the 16 subjects who could induce an eructation, the upper esophageal sphincter was partially visible or free for full inspection. Conclusion: The eructation method as a simple physiological function can be used as a complementary method during flexible transnasal endoscopy to enhance visibility of the entire hypopharynx region as well as the upper esophageal sphincter.

(C) 2019 The Author(s)

Published by S. Karger AG, Basel

\section{Introduction}

In routine medical examination, the hypopharyngeal-esophageal region (HER), which consists of the piriform fossa, the postcricoid space and the posterior pharyngeal wall, is difficult to assess due to its position, anatomical complexity, and constraints of space.

Flexible laryngeal endoscopy has become one of the most important imaging techniques for assessing this region [1]. Further development of the endoscopic technique like the narrow band imaging system are contributing to this $[2,3]$. Transnasal laryngoscopy is easy to use and safe, but its diagnostic value is determined mainly by the visibility of the hypopharyngeal structures, which is often incomplete, especially in the transition zone from the hypopharynx to the upper esophageal sphincter (UES).

Different authors describe various methods and their combined use aiming to improve the transnasal fiber-optic view of the HER. In particular, special positioning maneuvers such as head torsion [4], anterior neck skin traction [5], or the Killian position and its modified variants [6] have been described. Other procedures, some of which are also used in combination with the aforementioned maneuvers, attempt to dilate the anatomical structures of the hypopharynx with gas. Well-known examples are the trumpet maneuver and the Valsalva maneuver [7].

A similar approach is followed by the method presented in this work, in which a volitional eructation maneuver improves the endoscopic view of the HER including the UES. During this physiological process, the UES relaxes, allowing gas to escape from the stomach to decrease intra-abdominal pressure [8]. The larynx shifts in the anterior and cranial direction, the caudal hypopharyngeal area widens and the UES becomes visible. This approach provides insights into areas of the HER that are usually collapsed during flexible endoscopy.

The purpose of this study was to evaluate the feasibility of a volitional eructation maneuver during transnasal flexible laryngoscopy and its influence on the visibility of HER in a group of healthy volunteers.

\section{Materials and Methods}

In this study, we examined 20 healthy adult volunteers ( 7 males) from the age of 20 to 50 years. The subjects were examined in a normal sitting position. A regular flexible fiber endoscope (ORLVISION ${ }^{\circledR}$ RS1) was inserted. In order to minimize the impact on volunteers, xylometazoline (Otriven ${ }^{\circledR}$ ) was administered prior to the procedure in the lower nasal passage to prevent mucosal swelling during laryngeal endoscopy. In addition, lidocaine gel $2 \%\left(\right.$ Xylocain ${ }^{\circledR}$ ) was applied locally in the lower nasal passage as an analgesic.

Once the larynx was freely visible during laryngoscopy, subjects were asked to trigger a "burp." The volitional belching during the study was assisted by the previous gradual drinking of 150-330 mL of a carbonated cold drink as needed. 
Table 1. Grade of visualization of the hypopharynx (score according to Murono et al. [9])

\begin{tabular}{lll}
\hline Subjects, $n$ & Score & Description \\
\hline 0 & 1 & Only piriform sinuses are observed \\
3 & 2 & Part of postcricoid area is observed \\
0 & 3 & Whole postcricoid area is observed \\
1 & 4 & Greater than whole postcricoid area is observed \\
12 & 5 & Upper esophageal sphincter is observed \\
\hline 16 & 4.38 & Total/mean score \\
\hline
\end{tabular}

Table 2. Visibility of the HER by volitional eructation maneuver

\begin{tabular}{lccc}
\hline Subjects, $n$ & Piriform sinuses & Postcricoid area & Esophageal inlet \\
\hline Not visible & 0 & 0 & 4 \\
Partially visible & 1 & 3 & 7 \\
Complete view & 15 & 13 & 5 \\
Total & 16 & 16 & 16 \\
\hline
\end{tabular}

For later evaluation, the examination procedure was stored in a system for digital recording, postprocessing and archiving of medical film sequences and individual images (REHDER \& PARTNER rpSzene ${ }^{\circledR}$ ). The procedure was evaluated as a single image analysis. The best view of the HER during the belch was captured. Two independent evaluators blindly reviewed and graded those images according to a 5-point scale for the visualization of the HER.

In this graduation system developed by Murono et al. [9] in a previous study, score 1 was given if only the piriformis (PS) fossa was visible. Score 2 applied when a part of the postcricoid area (PC) was observed, but PC still has contact with the posterior pharyngeal wall (PW); score 3 when a space between PC and PW was confirmed and the whole PC could be observed. Score 4 was given when the space between PC and PW opened widely, but UES could not be clearly confirmed. Score 5 meant the UES was clearly observed. The average score was calculated for further analysis.

\section{Results}

In our group of 20 volunteers, 16 (80\%) were able to induce a volitional eructation during the laryngeal endoscopy. The expected laryngeal shift in the anterior and cranial direction and the hypopharyngeal extension were observed in all of these cases.

The average visibility score for the subjects who could perform the eructation maneuver during laryngeal endoscopy was calculated to be 4.38 (Table 1). In $13(81 \%)$ of the subjects, the UES was visible (score 4 or higher).

From the group of 16 subjects who were able to induce a belch, the hypopharyngeal structures with PS, PW, PC, and the fully opened UES were fully visible in $5(31 \%)$ on primary inspection (Table 2). In another 7 (44\%) of these subjects, the UES was visible, but only partially opened. For these total 12 (75\%) subjects, score 5 was given.

In the other 4 subjects, the space between PC and PW was wide open in $1(6 \%)$ case (score 4), and in 3 cases (19\%), PC was only partially visible (score 2).

The assessment of the visibility of PW, PC, and UES did not differ between the two reviewers (M.W. and D.S.). 


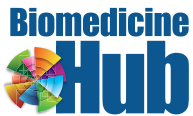

Fig. 1. The eructation maneuver as a physiological function during flexible laryngoscopy may allow full visibility of the hypopharynx as far as to the relaxed upper esophageal sphincter. \begin{tabular}{l} 
Biomed Hub 2019;4:495729 \\
\hline c) 2019 The Author(s). Published by S. Karger AG, Basel
\end{tabular} www.karger.com/bmh

Politz et al.: Enhancing Visibility of the Hypopharyngeal-Esophageal Region by Introduction of a Physiological Eructation Maneuver

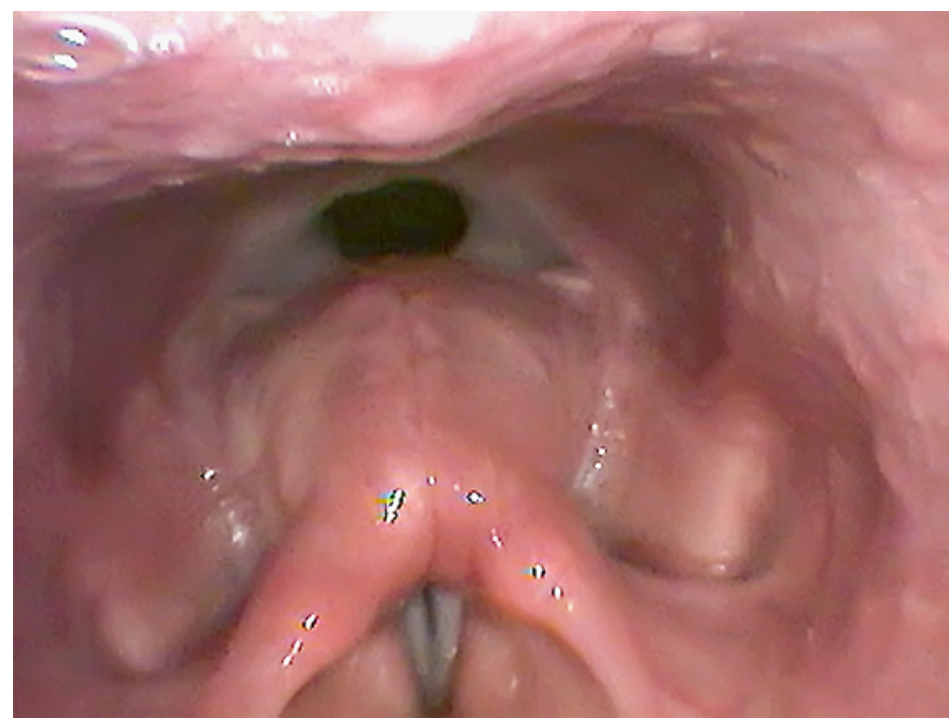

\section{Discussion}

We present observational data in a proof-of-concept approach to a new method for improving the visibility of hypopharyngeal structures through volitional belching during transnasal laryngoscopy. The hypopharyngeal esophageal region is difficult to assess with imaging techniques due to its anatomical characteristics. This increases the risk that diseases of this region such as superficial carcinomas in the early stages are recognized late $[10,11]$.

For this purpose, several procedures have been described for flexible transnasal hypopharyngoscopy to improve the view of the lower regions [12]. One possible approach is pneumatic dilatation using the trumpet maneuver or Valsalva maneuver during endoscopic examination. The UES is visible in up to $20 \%$ of patients using one of these procedures [7]. Both methods do not differ significantly in terms of their effectiveness. By using the trumpet maneuver in combination with anterior neck skin traction, the proportion of patients with visible UES can be increased to $40 \%$ [5].

Recent data show that this proportion of patients can be further increased up to $85 \%$ by a maneuver described by Sakai et al. $[6,13]$ as the modified Killian's method. For this method, Murono et al. [9] indicate a visibility score of up to 3.6 in a group of 57 patients who had been followed up for hypopharyngeal or laryngeal disease. This procedure combines self-insufflation with the Valsalva maneuver, head rotation, and a position described as the modified Killian's position with the neck bent to the front, depressed chin, and a bowed upper body.

With our method, the proportion of patients with UES visible during transnasal laryngoscopy was $81 \%$ (visibility score 4.38 ) when a belch could be triggered. The method thus leads to comparable results as the modified Killian's method. However, out of 20 subjects, 4 $(20 \%)$ could not volitionally trigger an eructation. Interestingly, in these 4 subjects, even the attempt to belch resulted in a slight laryngeal angulation and dilatation, so that in 1 case an area greater than the whole PC could be observed even without a belch (score 4).

In our method, we use a physiological function of the body, which, similar to other methods, expands the anatomical structures of the HER by means of a pneumatic dilatation and does not require any special positioning technique. In contrast to other procedures, in this noninvasive method, the UES is partially or even fully open due to the gas escaping from the stomach (Fig. 1). As with the flexible endoscopic assessment of swallowing, the evaluation is performed with a single image analysis (50 images/s) after the examination, which allows 
the assessment of the hypopharyngeal mucosa, although the duration of the belch during the examination is short. Technical innovations such as narrow band imaging endoscopy can further enhance the validity of such an examination. This could potentially help to better select patients for more invasive procedures such as biopsy requiring laryngoscopy under general anesthesia.

The use of our method is limited by the fact that not all patients can volitionally trigger a belch, even if this is supported by the swallowing of a carbonated cold drink. The procedure, which has so far only been tested on a small group of volunteers, should be further investigated in a sufficiently large group of patients to evaluate our observations in a next step. Another limitation is the lack of direct comparison to other methods aiming at an improved visibility of the area under question and that only healthy volunteers were included.

We see our method most likely as a complementary technique to previously known methods. As a physiological body function, it can be used without significant risks and without much additional effort. If successful, the method improves the laryngoscopic view of the HER in a clinically meaningful way, providing partial or even full visibility of the UES.

\section{Conclusion}

In this observational study, $80 \%$ of healthy volunteers were able to volitionally produce an eructation during transnasal laryngoscopy enhancing visibility of the hypopharyngealesophageal transition zone. The eructation method as a simple physiological function can be used during flexible transnasal endoscopy to obtain a view of the entire hypopharynx region as well as the UES. The results from this observational study should be reproduced and expanded in patients or subjects at risk for hypopharyngeal cancer.

\section{Statement of Ethics}

Informed consent was obtained from all individual participants included in the study. Swissethics: the study has been registered under the project ID: PB_2017-00311. Old reference number before BASEC: BASEC-Nr.2006-01592. Ethical approval: "All procedures performed in studies involving human participants were in accordance with the ethical standards of the institutional and/or national research committee and with the 1964 Helsinki declaration and its later amendments or comparable ethical standards."

\section{Disclosure Statement}

The authors have no conflicts of interest to declare.

\section{Funding Sources}

There are no funding sources. 


\section{Author Contributions}

Svetlana Politz: study concept and design, acquisition of data, analysis and interpretation, draft of the manuscript.

Martin Wagner: analysis and interpretation, critical revision of the manuscript for important intellectual content, editing of the article before submission.

Degenhart Schwub: acquisition of data, analysi,s and interpretation.

Sandra Cattenberg: acquisition of data, analysis, and interpretation.

Robert Thurnheer: critical revision of the manuscript for important intellectual content, review and editing of the article before submission.

Ludwig Schelosky: study supervision, critical revision of the manuscript for important intellectual content, editing of the article before submission.

\section{References}

1 Tsikoudas A, Mochloulis G. Role of transnasal oesophagoscopy in diagnosis of early malignancy in the area of the oesophagus and hypopharynx. A review of the literature. Eur Arch Otorhinolaryngol. 2014 Jan;271(1): 203-4.

2 Muto M, Nakane M, Katada C, Sano Y, Ohtsu A, Esumi H, et al. Squamous cell carcinoma in situ at oropharyngeal and hypopharyngeal mucosal sites. Cancer. 2004 Sep;101(6):1375-81.

3 Zhou H, Zhang J, Guo L, Nie J, Zhu C, Ma X. The value of narrow band imaging in diagnosis of head and neck cancer: a meta-analysis. Sci Rep. 2018 Jan;8(1):515.

4 Tsunoda A, Ishihara A, Kishimoto S, Tsunoda R, Tsunoda K. Head torsion technique for detailed observation of larynx and hypopharynx. J Laryngol Otol. 2007 May;121(5):489-90.

5 Colquhoun-Flannery W, Davis A, Carruth JA. Improving the endoscopic view of the hypopharynx with anterior neck traction during the trumpet manoeuvre. J Laryngol Otol. 2000 Apr;114(4):283-4.

6 Sakai A, Okami K, Sugimoto R, Ebisumoto K, Yamamoto H, Maki D, et al. A new technique to expose the hypopharyngeal space: the modified Killian's method. Auris Nasus Larynx. 2014 Apr;41(2):207-10.

7 Freeman SR, Keith AO, Aucott W, Kazmi N, Nigam A. Comparison between two valsalva techniques for improvement of hypopharyngeal nasendoscopy: a preliminary communication. Clin Otolaryngol. 2007 Dec; 32(6):488-91.

8 Bredenoord AJ, Smout AJ. Physiologic and pathologic belching. Clin Gastroenterol Hepatol. 2007 Jul;5(7): 772-5.

9 Murono S, Tsuji A, Endo K, Kondo S, Wakisaka N, Yoshizaki T. Evaluation of modified Killian's method: a technique to expose the hypopharyngeal space. Laryngoscope. 2014 Nov;124(11):2526-30.

10 Warnakulasuriya S. Global epidemiology of oral and oropharyngeal cancer. Oral Oncol. 2009 Apr-May;45(45):309-16.

11 Wycliffe ND, Grover RS, Kim PD, Simental A Jr. Hypopharyngeal cancer. Top Magn Reson Imaging. 2007 Aug; 18(4):243-58.

12 Williams RS, Lancaster J, Karagama Y, Tandon S, Karkanevatos A. A systematic approach to the nasendoscopic examination of the larynx and pharynx. Clin Otolaryngol Allied Sci. 2004 Apr;29(2):175-8.

13 Sakai A, Okami K, Ebisumoto K, Yamamoto H, Saito K, Maki D, et al. How to improve the endoscopic view of the hypopharynx: validation of our novel modified Killian's Method to expose the hypopharynx in 20 subjects. Clin Otolaryngol. 2018 Oct;43(5):1407-10. 